\title{
Brain expression of the vascular endothelial growth factor gene family in cognitive aging and alzheimer's disease
}

\author{
Emily R. Mahoney ${ }^{1,2} \cdot$ Logan Dumitrescu ${ }^{1,2} \cdot$ Annah M. Moore ${ }^{1,2} \cdot$ Francis E. Cambronero ${ }^{1} \cdot$ Philip L. De Jager $\mathbb{D}^{3,4}$. \\ Mary Ellen I. Koran ${ }^{5}$ Vladislav A. Petyuk ${ }^{6}{ }^{6} \cdot$ Renã A. S. Robinson $^{7} \cdot$ Sandeep Goyal $^{7} \cdot$ Julie A. Schneider $^{8}$. \\ David A. Bennett ${ }^{8}$ - Angela L. Jefferson ${ }^{1} \cdot$ Timothy J. Hohman (D) $^{1,2}$
}

Received: 11 January 2019 / Revised: 19 April 2019 / Accepted: 17 May 2019 / Published online: 22 July 2019

(c) The Author(s) 2019. This article is published with open access

\begin{abstract}
Vascular endothelial growth factor (VEGF) is associated with the clinical manifestation of Alzheimer's disease (AD). However, the role of the VEGF gene family in neuroprotection is complex due to the number of biological pathways they regulate. This study explored associations between brain expression of VEGF genes with cognitive performance and AD pathology. Genetic, cognitive, and neuropathology data were acquired from the Religious Orders Study and Rush Memory and Aging Project. Expression of ten VEGF ligand and receptor genes was quantified using RNA sequencing of prefrontal cortex tissue. Global cognitive composite scores were calculated from 17 neuropsychological tests. $\beta$-amyloid and tau burden were measured at autopsy. Participants $(n=531)$ included individuals with normal cognition $(n=180)$, mild cognitive impairment $(n=148)$, or AD dementia $(n=203)$. Mean age at death was 89 years and $37 \%$ were male. Higher prefrontal cortex expression of VEGFB, FLT4, FLT1, and PGF was associated with worse cognitive trajectories ( $p \leq 0.01$ ). Increased expression of $V E G F B$ and FLT4 was also associated with lower cognition scores at the last visit before death $(p \leq$ 0.01). VEGFB, FLT4, and FLT1 were upregulated among AD dementia compared with normal cognition participants ( $p \leq$ 0.03 ). All four genes associated with cognition related to elevated $\beta$-amyloid ( $p \leq 0.01)$ and/or tau burden $(p \leq 0.03)$. VEGF ligand and receptor genes, specifically genes relevant to FLT4 and FLT1 receptor signaling, are associated with cognition, longitudinal cognitive decline, and AD neuropathology. Future work should confirm these observations at the protein level to better understand how changes in VEGF transcription and translation relate to neurodegenerative disease.
\end{abstract}

\section{Introduction}

Vascular endothelial growth factors (VEGFs) are important signaling proteins involved in the growth and maintenance

Supplementary information The online version of this article (https:// doi.org/10.1038/s41380-019-0458-5) contains supplementary material, which is available to authorized users.

$\square$ Timothy J. Hohman

Timothy.J.Hohman@vumc.org

1 Vanderbilt Memory and Alzheimer's Center, Vanderbilt University Medical Center, Nashville, TN, USA

2 Vanderbilt Genetics Institute, Vanderbilt University Medical Center, Nashville, TN, USA

3 Center for Translational \& Computational Neuroimmunology, Department of Neurology, Columbia University Medical Center, New York, NY, USA of both vascular and neural cells [1]. VEGFA, the founding and most studied member of the VEGF family, appears to protect against cognitive impairment [2], particularly in the context of Alzheimer's disease (AD) pathology [2-4]. However, the role of VEGFA in neurodegenerative disease is quite complex. In the context of $\mathrm{AD}$ dementia, there is mixed evidence in the literature for both up- and downregulation of VEGFA gene and protein expression in the brain, blood, and cerebrospinal fluid (CSF). For example,

4 Cell Circuits Program, Broad Institute, Cambridge, MA, USA

5 Stanford Hospital, Department of Radiology, Stanford, CA, USA

6 Biological Sciences Division, Pacific Northwest National Laboratory, Richland, WA, USA

7 Department of Chemistry, Vanderbilt University, Nashville, TN, USA

8 Rush Alzheimer's Disease Center, Rush University Medical Center, Chicago, IL, USA 
protein levels of VEGFA have been reported to be higher among AD dementia cases compared with controls in CSF [5], plasma [6], and the medial parietal cortex [7]. Moreover, higher VEGF levels in the AD brain have been associated with loss of pericytes [8], increased blood-brain barrier permeability [8], and more severe tangle pathology [9].

Yet, there is also evidence of downregulation of VEGFA in the context of $\mathrm{AD}$ pathology, depending on the brain region sampled and the isoform of VEGFA. For example, one study observed higher $\mathrm{VEGF}_{189}$ levels and lower $\mathrm{VEGF}_{121}$ levels in the hippocampus of AD cases compared with controls [10]. Beyond tissue expression levels in the brain, there is also evidence of fewer VEGFA-positive capillaries in AD brains compared with controls [11], and an association between lower levels of ventricular fluid VEGF protein levels and a higher tangle burden at autopsy [12]. Additional evidence outside of the brain also suggests that VEGFA levels may be lower in both serum and CSF among $\mathrm{AD}$ cases compared with controls [4, 13]. In sum, the literature supports VEGFA alterations over the course of the AD neuropathological cascade, but the exact association appears to depend on the tissue, isoform, and stage of disease at which the sample is acquired.

Despite the complexity and mixed evidence of VEGF associations with $\mathrm{AD}$ dementia, there is growing evidence that VEGF may have a neuroprotective role. For example, work from our group has indicated that higher-baseline CSF VEGFA levels are associated with slower rates of hippocampal atrophy and slower rates of cognitive decline [3], particularly among individuals with elevated levels of AD biomarkers. Mouse models of AD offer additional support for the neuroprotective role of VEGFA, as memory deficits were halted when animals were treated with VEGFA $[2,14,15]$.

The challenge in better characterizing VEGF alterations during brain aging and $\mathrm{AD}$ dementia may be due to the large number of biological pathways differentially influenced by distinct VEGF signaling proteins. The mammalian VEGF protein family includes five ligands (VEGFA, VEGFB, VEGFC, VEGFD, and PGF [placental growth factor]), three receptors (FLT1 [also called VEGFR1], KDR [also called VEGFR2], and FLT4 [also called VEGFR3]), and two co-receptors (NRP1 and NRP2), each with multiple isoforms. These ligands and receptors interact to exert different downstream effects [1]. For example, while it has been suggested that VEGFB signaling through the FLT1 receptor can mediate neuroprotection through NMDA alterations [16, 17], KDR and FLT4 signaling have been hypothesized to mediate angiogenesis through AKT signaling pathways and activation of transcription factors $[1,18]$. Comprehensive assessment of VEGF ligands and receptors in the context of cognitive aging may provide clues as to which VEGF signaling pathways are most relevant to neuroprotection.

The aim of this study was to examine associations between prefrontal cortex expression of VEGF ligand and receptor genes in relation to cognitive performance, $A D$ dementia, and postmortem AD neuropathology. We hypothesized that expression of $V E G F$ genes in the brain would differentially relate to cognitive performance and $\mathrm{AD}$ pathology.

\section{Materials and methods}

\section{Participants}

Data were acquired from two well-characterized cohort studies of aging and dementia. The Religious Orders Study (ROS), begun in 1994, and the Rush Memory and Aging Project (MAP), begun in 1997, involve older adults who enrolled without dementia, agreed to annual clinical evaluations and organ donation at death, and signed an Anatomical Gift Act for brain donation [19-21]. Written informed consent was obtained from participants, and research was carried out in accordance with Institutional Review Board (IRB)-approved protocols. Secondary analyses of all data were approved by the Vanderbilt University Medical Center IRB. ROSMAP data are available online at the Rush Alzheimer's Disease Center Resource Sharing Hub (https://www.radc.rush.edu/), as well as on the Accelerating Medicines Partnership-Alzheimer's Disease (AMP-AD) Knowledge Portal (syn3219045).

\section{Neuropsychological composites}

Composite measures of cognition have been calculated in ROS/MAP [22]. Briefly, global cognition was derived from a total of 17 tests across five domains of cognition (episodic, semantic, and working memory, perceptual orientation, and perceptual speed). A global cognition composite was made by averaging the $z$-scores of all available tests.

\section{Autopsy measures of VEGF gene expression}

RNA expression levels were obtained from frozen sections of the dorsolateral prefrontal cortex that were manually dissected from postmortem brain tissue. Details of RNA extraction, processing, quality control, and normalization have been published [23]. Briefly, RNA was isolated using the RNeasy lipid tissue kit (Qiagen, Valencia, CA) and was reverse transcribed and biotin-UTP labeled using the llumina TotalPrep ${ }^{\mathrm{TM}}$ RNA Amplification Kit from Ambion ${ }^{\mathrm{TM}}$ (Illumina, San Diego, CA). Expression signals were generated using the BeadStudio software suite (Illumina, San 
Diego, CA). Standard control and normalization methods were employed to account for technical variability, due to differences in hybridization dates. Expression levels of ten $V E G F$ ligand and receptor genes with 63 available isoforms were analyzed (number of isoforms in parenthesis): VEGFA (14), VEGFB (3), VEGFC (2), VEGFD (2), NRPI (12), NRP2 (13), FLT1 (3), FLT4 (8), KDR (1), and PGF (5). Primary analyses focused on gene count data quantified using RSEM. Reads were aligned using the Bowtie 1 package and then counted using RSEM. Secondary analyses assessed isoform abundance, which was also quantified using RSEM. Low-abundance isoforms (expressed in < $10 \%$ of the cohort) were filtered out from analyses to reduce confounding due to floor effects. Outliers (values 4 standard deviations above or below the combined sample mean) were also removed.

\section{Measures of neural and cerebrovascular pathology}

All measures of pathology were characterized previously in ROS/MAP $[19,20]$. $\beta$-amyloid and tau neuropathologies were measured in two ways: immunohistochemistry and microscopic evaluation of silver-stain crosssections. The percentage of area occupied by $\beta$-amyloid or tau was an average of anti-A $\beta$ and anti-phosphorylated tau immunohistochemistry evaluation from eight regions (hippocampus, angular gyrus, and entorhinal, midfrontal, inferior temporal, calcarine, anterior cingulate, and superior frontal cortices). Counts of neuritic plaques and neurofibrillary tau tangles (NFT) were evaluated on silver-stained slides from five regions (hippocampus and entorhinal, midtemporal, inferior parietal, and midfrontal cortices). These counts were entered as continuous variables and were transformed prior to analysis to better approximate a normal distribution.

TDP-43 pathology was analyzed in six regions (amygdala, hippocampus CA1, dentate gyrus, entorhinal, midtemporal, and midfrontal cortices) by staining with monoclonal antibodies to phosphorylated TDP-43 and was scored from 0 (no pathology) to 4 (presence of pathology in all regions) [24]. Measurement details for cerebral amyloid angiopathy (CAA), atherosclerosis, arteriolosclerosis, and gross- and micro-infarcts have been published [25-31]. Briefly, CAA was measured in the midfrontal, midtemporal, angular, and calcarine cortices and scored from 0 to 3 , with 3 being the highest deposition level $[27,29]$. Atherosclerosis was measured by visual inspection of circle of Willis vessels and given a score from 0 (no significant atherosclerosis) to 3 (over half had atherosclerosis or at least one had $75 \%$ occlusion or both) [25]. Arteriolosclerosis was measured histologically and classified into four stages, 0 indicating no changes and 3 indicating severe changes [28]. Gross infarcts were measured from nine regions (midfrontal, middle temporal, entorhinal, hippocampal, inferior parietal and anterior cingulate cortices, anterior basal ganglia, thalamus, and midbrain) by visual inspection and confirmed histologically. Micro-infarcts were examined from the same nine regions by $6-\mu \mathrm{m}$ paraffinembedded sections stained with hematoxylin/eosin. Both were given a binary score, indicating the presence or absence of infarcts [26, 30, 31]. In addition, total count and volume of macro-infarcts were assessed by visual inspection to quantify the extent of ischemic brain damage.

\section{Differential expression replication data sets}

Data from two additional cohorts from the AMP-AD Knowledge Portal (syn14237651), the MayoRNAseq study (syn5550404), and the Mount Sinai Brain Bank (MSBB) study (syn3159438), were leveraged for replication of differential expression results. For the Mayo cohort, postmortem samples were collected from the temporal cortex and cerebellum, as previously described [32, 33]. For the MSBB cohort, postmortem samples were collected from the parahippocampal gyrus, frontal pole, superior temporal gyrus, and inferior frontal gyrus, as previously described [34]. Clinical diagnosis was harmonized between the studies using Braak staging, tau pathology, and cognitive scores.

\section{Statistical analyses}

Statistical analyses were completed using $\mathrm{R}$ (versions 3.4.3 and 3.5.0; https://www.r-project.org/) and code is available on request from the authors. Significance was set a priori to $\alpha=0.05$. $P$-value corrections were performed using the false discovery rate (FDR) method. All models were restricted to individuals who had their last cognitive assessment within 2 years of death. For all analyses, each gene was run individually.

Prior to the main analyses, we assessed differences in $V E G F$ expression among diagnostic groups using linear regression models, covarying for age at death and sex. In replication data sets, weighted mixed-effect linear models with sex and age of death as fixed effects and donor as a random effect were used to analyze differences in gene expression between participants with $\mathrm{AD}$ and controls in each study. $P$-value corrections were made using the FDR procedure, correcting for all genes analyzed (between 16,348 and 18,520 depending on the tissue). Normalization and covariate adjustments were performed for each study separately to account for differences between studies.

For the main analysis, we evaluated associations between $V E G F$ expression levels in prefrontal cortex tissue with 
Table 1 Participant characteristics

\begin{tabular}{|c|c|c|c|c|c|}
\hline & \multicolumn{3}{|l|}{ Clinical diagnosis } & \multirow[t]{2}{*}{ Total (531) } & \multirow[t]{2}{*}{$P$} \\
\hline & Normal cognition (180) & $\begin{array}{l}\text { Mild cognitive } \\
\text { impairment (148) }\end{array}$ & $\begin{array}{l}\text { Alzheimer's } \\
\text { disease (203) }\end{array}$ & & \\
\hline Age of death, years & $86 \pm 7$ & $89 \pm 6$ & $91 \pm 6$ & $89 \pm 7$ & $<0.001$ \\
\hline Male, no. $(\%)$ & $70(39)$ & $54(36)$ & $70(34)$ & $194(37)$ & 0.67 \\
\hline Non-Hispanic white, no. (\%) & $177(98)$ & $146(99)$ & $195(96)$ & $518(98)$ & 0.21 \\
\hline Education, years & $17 \pm 4$ & $16 \pm 3$ & $17 \pm 4$ & $17 \pm 4$ & 0.59 \\
\hline $\begin{array}{l}\text { Global cognition composite (at the } \\
\text { last visit), } \mathrm{z}\end{array}$ & $0.14 \pm 0.42$ & $-0.49 \pm 0.45$ & $-1.85 \pm 0.91$ & $-0.80 \pm 1.09$ & $<0.001$ \\
\hline Average number of visits & $7.12 \pm 4.04$ & $6.93 \pm 3.65$ & $7.55 \pm 3.69$ & $7.23 \pm 3.8$ & 0.26 \\
\hline
\end{tabular}

Boldface signifies $p<0.05$
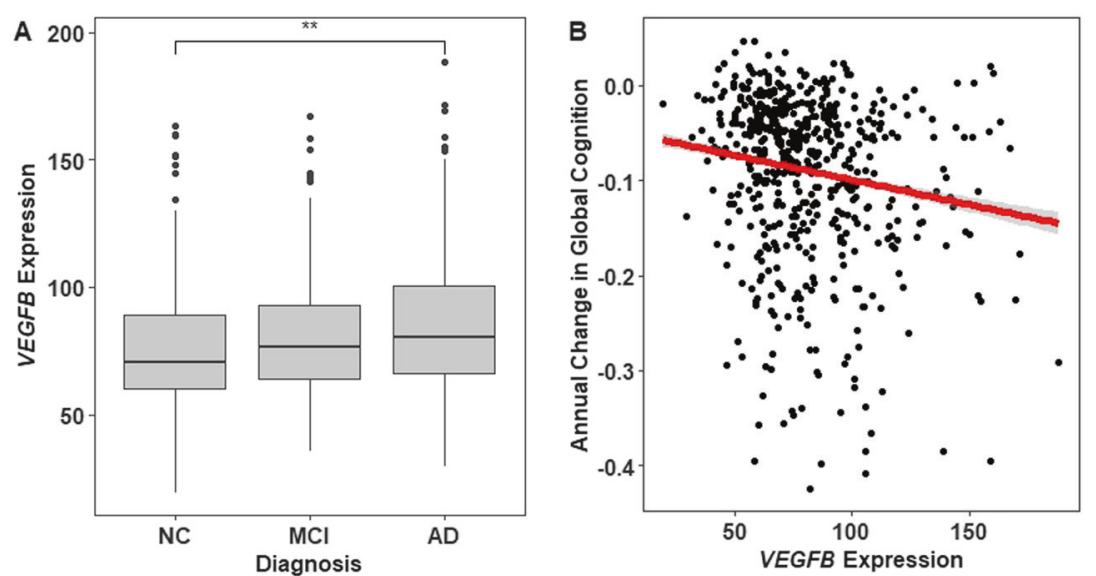

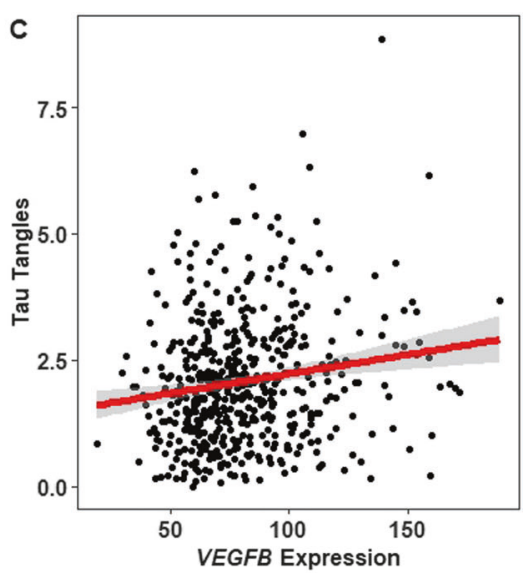

Fig. 1 VEGFB associations with longitudinal cognition, AD dementia, and tau pathology. Prefrontal cortex expression of $V E G F B$ (a) differed between participants with $\mathrm{AD}$ dementia compared with those with normal cognition, $\mathbf{b}$ was negatively associated with longitudinal global

cognition, and $\mathbf{c}$ was positively associated with tau burden at autopsy. **p-value $<0.01$; NC normal cognition, MCI mild cognitive impairment, AD Alzheimer's disease

global cognition cross-sectionally (at the final visit prior to death) as well as longitudinally (over the years preceding death). Longitudinal associations were evaluated using mixed-effect regression with a gene expression $\times$ interval interaction term, with interval modeled as time in years before death. Age at death, sex, gene expression level, and interval were entered as fixed effects with the intercept and interval entered as random effects. The global cognition composite was entered as a continuous outcome. Crosssectional associations were evaluated using linear regression covarying for age at death, sex, and interval between the last visit and death. Secondary analyses analyzed diagnostic interactions and assessed isoform abundance data to clarify the most relevant isoforms of each gene.

To remove possible effects of sample differences in celltype composition across age and disease, proportions of neurons, microglia, oligodendrocytes, and endothelial cells were estimated using the prefrontal cortex expression of known cell-specific marker genes: ENO2 (neurons), CD68 (microglia), OLIG2 (oligodendrocytes), GFAP (astrocytes), and CD34 (endothelial cells). Correlations between VEGF genes and cell-specific markers were calculated using Pearson's R. Next, models were rerun covarying for ENO2 in one model and again covarying for all other cell types in a second model. In addition, we residualized the association between a given gene and cell marker to create a corrected expression score and analyzed the models again.

Last, we evaluated associations between VEGF expression levels and AD neuropathology. Associations with continuous outcomes of $\beta$-amyloid, tau, neuritic plaques, and NFT were analyzed using linear models. Secondary analyses assessed associations between VEGF expression and non-AD neuropathology. Associations with multilevel categorical outcomes (CAA, atherosclerosis, arteriolosclerosis, and TDP-43) were analyzed with proportionalodds logistic regression. Associations with binary categorical outcomes were analyzed with binary logistic regression. Associations with gross-infarct count and volume were assessed using Poisson regression. All models covaried for age at death and sex. 
Table 2 VEGF associations with global cognition

\begin{tabular}{|c|c|c|c|c|c|c|c|c|}
\hline \multirow[b]{2}{*}{ Gene } & \multicolumn{4}{|c|}{ Longitudinal } & \multicolumn{4}{|c|}{ Cross-sectional } \\
\hline & $\beta$ & $\mathrm{SE}$ & $P$ & P.fdr & $\beta$ & $\mathrm{SE}$ & $P$ & P.fdr \\
\hline$V E G F B$ & -0.001 & 0.0002 & $5.66 \mathrm{E}-05$ & 0.001 & -0.006 & 0.002 & 0.001 & 0.006 \\
\hline FLT4 & -0.030 & 0.009 & $4.47 \mathrm{E}-04$ & 0.002 & -0.207 & 0.078 & 0.008 & 0.040 \\
\hline FLT1 & -0.004 & 0.001 & 0.002 & 0.005 & -0.025 & 0.012 & 0.038 & 0.126 \\
\hline$P G F$ & -0.010 & 0.003 & 0.002 & 0.005 & -0.051 & 0.028 & 0.075 & 0.187 \\
\hline$N R P 1$ & 0.004 & 0.004 & 0.279 & 0.484 & 0.054 & 0.036 & 0.133 & 0.265 \\
\hline$N R P 2$ & 0.004 & 0.007 & 0.582 & 0.646 & 0.073 & 0.066 & 0.273 & 0.455 \\
\hline$V E G F C$ & -0.017 & 0.018 & 0.339 & 0.484 & -0.128 & 0.164 & 0.437 & 0.546 \\
\hline VEGFD & -0.006 & 0.015 & 0.693 & 0.693 & -0.109 & 0.139 & 0.436 & 0.546 \\
\hline$V E G F A$ & -0.001 & 0.001 & 0.302 & 0.484 & -0.003 & 0.005 & 0.493 & 0.547 \\
\hline$K D R$ & -0.007 & 0.012 & 0.540 & 0.646 & 0.044 & 0.113 & 0.697 & 0.697 \\
\hline
\end{tabular}

Boldface signifies corrected P.fdr $<0.05$

P.fdr column contains $p$-values corrected for ten tests using the false discovery rate (FDR)

Table 3 VEGF associations with AD pathology

\begin{tabular}{|c|c|c|c|c|c|c|c|c|c|c|c|c|c|c|c|c|}
\hline \multirow[b]{2}{*}{ Gene } & \multicolumn{4}{|c|}{$\beta$-amyloid } & \multicolumn{4}{|l|}{ Tau } & \multicolumn{4}{|c|}{ Neuritic plaques } & \multicolumn{4}{|c|}{ Neurofibrillary tangles } \\
\hline & $\beta$ & SE & $P$ & P.fdr & $\beta$ & SE & $P$ & P.fdr & $\beta$ & SE & $P$ & P.fdr & $\beta$ & SE & $P$ & P.fdr \\
\hline FLT1 & 0.045 & 0.013 & 0.001 & 0.002 & 0.022 & 0.014 & 0.122 & 0.122 & 0.009 & 0.006 & 0.136 & 0.136 & 0.006 & 0.004 & 0.193 & 0.193 \\
\hline FLT4 & 0.243 & 0.084 & 0.004 & 0.008 & 0.228 & 0.091 & 0.013 & 0.017 & 0.078 & 0.038 & 0.040 & 0.053 & 0.053 & 0.028 & 0.056 & 0.168 \\
\hline$P G F$ & 0.060 & 0.031 & 0.049 & 0.066 & 0.107 & 0.033 & 0.001 & 0.003 & 0.034 & 0.014 & 0.014 & 0.028 & 0.015 & 0.010 & 0.126 & 0.168 \\
\hline$V E G F B$ & 0.002 & 0.002 & 0.190 & 0.190 & 0.007 & 0.002 & 0.001 & 0.003 & 0.002 & 0.001 & 0.010 & 0.028 & 0.001 & 0.001 & 0.113 & 0.168 \\
\hline
\end{tabular}

Boldface signifies corrected P.fdr $<0.05$

P.fdr column contains $p$-values corrected for four tests using the false discovery rate (FDR)

\section{Results}

Participant characteristics are presented in Table 1. Participants were mostly well-educated, non-Hispanic white, and had diagnoses of normal cognition, mild cognitive impairment, and $\mathrm{AD}$ dementia. As expected, global cognition scores differed significantly across diagnostic groups $(p<$ 0.001). Participants with AD dementia had an older mean age of death compared with the other groups $(p<0.001)$.

\section{Diagnostic differences in VEGF expression}

$V E G F B$ was expressed at higher levels in $\mathrm{AD}$ dementia as compared with normal cognition participants (corrected $p$ $=0.04$; Fig. 1b), a finding which replicated in both the Mayo (cerebellum and temporal cortex tissues) and MSBB cohorts (parahippocampal gyrus). Differences across all three diagnostic groups for the whole VEGF family are presented in Supplementary Table 1. In addition, we observed nominal increased expression between participants with neuropathologically confirmed AD compared with controls in FLT1 and FLT4 (corrected $p>0.05, p<$ 0.01; Supplementary Figs. $1 \mathrm{~A}$ and 2A), which replicated among Mayo participants in the temporal cortex for
FLT4 (corrected $p=0.006$ ) and in both the cerebellum and temporal cortex for FLT1 (corrected $p<0.02$ ). See Supplementary Tables 2 and 3 for differential expression results for the whole $V E G F$ family in the AMP-AD cohorts.

\section{Associations with cognition}

Longitudinal and cross-sectional associations with global cognition are presented in Table 2. In cross-sectional analyses, increased VEGFB and FLT4 expression levels were associated with worse cognition. In longitudinal analyses, higher levels of ligand genes $V E G F B$ and $P G F$ and receptor genes FLT1 and FLT4 were associated with worse cognitive trajectories (Fig. 1b, Supplementary Figs. 3A, 1B, and 2B). In sensitivity analyses covarying for diagnosis (Supplementary Table 4), all longitudinal associations remained significant $(p \leq 0.004)$, and of the two cross-sectional associations, $V E G F B$ remained significant $(p=0.047)$ while FLT4 did not $(p=0.391)$. In addition, no interactions were observed between diagnosis and gene expression in predicting cognition cross-sectionally or longitudinally after correction for multiple tests (Supplementary Table 5). In isoform sensitivity analyses (Supplementary Table 6), all four $V E G F$ genes associated with cognition showed two or 
Fig. 2 Associations in the VEGF family with cognition, $\mathrm{AD}$ dementia, and AD pathology. This figure presents an illustration of the VEGF family, with arrows drawn from each ligand to each receptor/coreceptor with which it binds. Gene expression levels of the ligands and receptors in color (VEGFB, PGF, FLT4, and FLT1) were associated with worsening cognitive performance prior to death and associated with amyloid or tau pathology at autopsy. Asterisk indicates genes that were expressed differently between participants with $\mathrm{AD}$ dementia and those with normal cognition

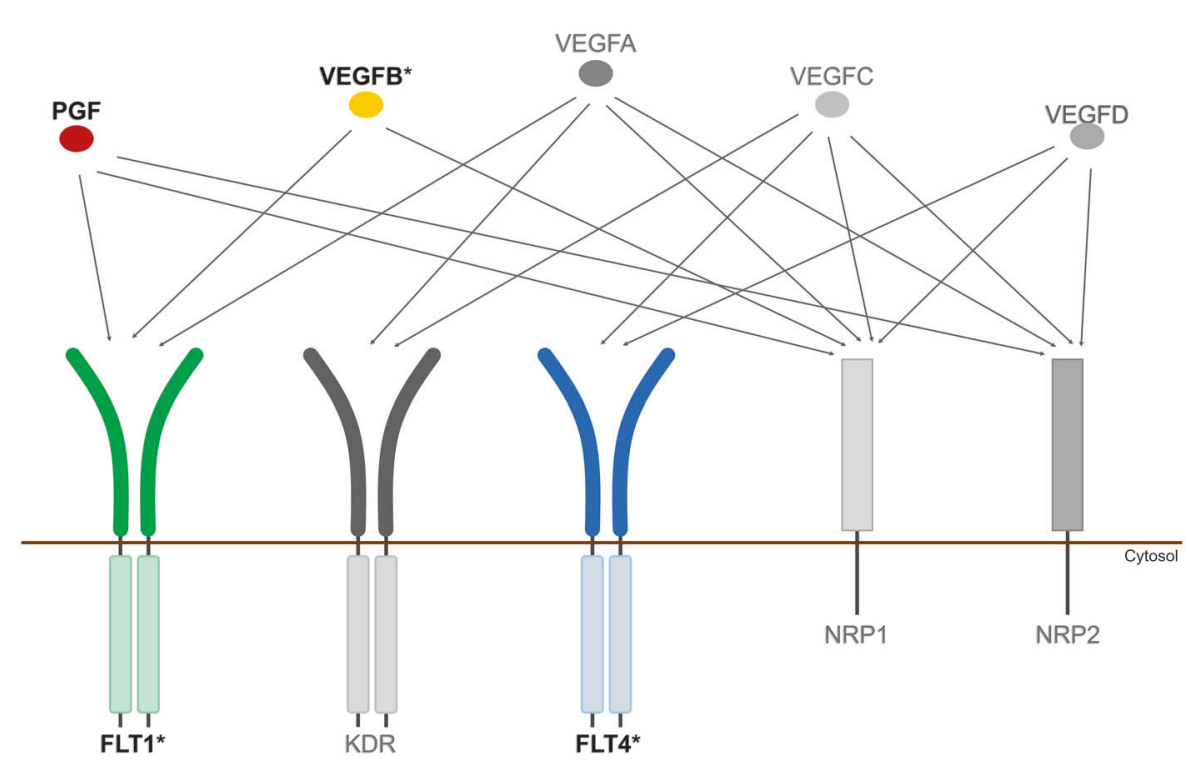

more isoform-specific associations comparable with the gene-level results.

Several genes were correlated with expression of cellspecific markers (see Supplementary Fig. 4 for correlation matrix). Specifically, VEGFB, FLT4, and PGF showed strong negative correlations $(r<-0.55)$ with ENO2 expression, a marker for neurons. $V E G F B$ also showed a strong positive correlation $(r=0.59)$ with $O L I G 2$ expression, a marker for oligodendrocytes. Although crosssectional associations were attenuated when adjusting for cell-specific markers of neurons (see Supplementary Table 7), longitudinal results remained largely unchanged, regardless of the type of cell-specific adjustment applied (see Supplementary Table 8).

\section{Associations with pathology}

Next, we tested for associations between the four VEGF genes that showed significant associations with cognition (VEGFB, FLT1, FLT4, and PGF) and neuropathology. The results for AD pathology are presented in Table 3. In analyses of $\beta$-amyloid burden, we observed associations with FLT1, FLT4, and PGF (see Supplementary Figs. 1B, 2B, and $3 \mathrm{~B}$ ). In analyses of tau burden, we observed associations with $V E G F B, F L T 4$, and $P G F$ (Fig. 1c, Supplementary Figs. 2D and 3D). VEGFB, PGF, and FLT4 were also associated with neuritic plaques. No associations were observed with NFT. See Supplementary Table 9 for associations between the six VEGF genes, which had no associations with cognition and AD pathology. In analyses of non-AD pathology, $V E G F B$ was positively associated with atherosclerosis $(\beta=0.006, \mathrm{SE}=0.003$, and $p=0.039)$ and arteriolosclerosis $(\beta=0.008, \mathrm{SE}=0.003$, and $p=0.013$; see Supplementary Table 10). VEGFB and FLT1 were associated with increased infarct count $(p<0.005)$ and volume $(p<0.035$; see Supplementary Table 11). See Supplementary Table 12 for isoform associations with pathology.

Taking all these results together, the model in Fig. 2 illustrates how the VEGF ligands and receptors interact, and which components are associated with cognition, $\mathrm{AD}$ dementia, and AD pathology.

\section{Discussion}

Prefrontal cortex expression of multiple VEGF genes, including ligand genes $V E G F B$ and $P G F$ and receptor genes $F L T 1$ and FLT4, were associated with a more rapid rate of cognitive decline late in life, with VEGFB and FLT4 also associated with cognitive scores closest to death. In addition, higher expression levels of these genes were observed among individuals with $\mathrm{AD}$ dementia, a finding which replicated across multiple data sets, and were associated with increased AD neuropathology at autopsy. These results suggest that alterations along VEGF signaling pathways occur with AD-related decline and are relevant to the cognitive progression of the disease.

We observed associations between prefrontal cortex expression of $F L T 1$ and its ligand genes VEGFB and $P G F$ in relation to longitudinal cognitive performance, with $V E G F B$ also associated with cognition at the last visit prior to death. While VEGFA-KDR signaling is the classical angiogenic pathway, FLT1 is known to have dual roles in neurogenesis and angiogenesis. Membrane-bound FLT1 signaling has been well-characterized in peripheral vascular cells where it is known to facilitate inflammatory cell recruitment [35], but the recent recognition that FLT1 may be predominantly expressed in neurons [36] and glia [37] in $\mathrm{AD}$ brains raises the question of unidentified roles 
for this receptor in neural repair processes, particularly under conditions of systemic hypoxia. For example, VEGFB has been shown to downregulate cell death-related signaling pathways in neurons through tyrosine-kinase activity of FLT1 [38, 39]. Thus, FLT1-mediated VEGFB signaling appears to promote neuroprotection (rather than acting as an angiogenic factor) in injury models of the nervous system. In support of this, we observed increased ischemic damage associated with higher expression of $V E G F B$ and FLT1. In the present analysis, associations between $V E G F B$ and faster cognitive decline may therefore reflect a downstream neural repair response to $\mathrm{AD}$ pathology.

FLT1 may also promote angiogenesis through PGF signaling. Although both VEGFB and PGF bind to FLT1, PGF appears to have stronger angiogenic effects compared with VEGFB, inducing neurovascular repair and the formation of perfused arterialized microvessels [40]. In our analyses, higher $P G F$ expression related to worse clinical outcomes, potentially reflecting a proangiogenic state coinciding with AD pathology that may be beneficial or detrimental to brain health. However, from the present data it is difficult to determine whether the observed genetic associations are the cause or consequence of disease.

The mixed accounts of FLT1's role in angiogenesis are partially clarified by noting the distinct roles that its isoforms play. Membrane-bound FLT1 is the signaling subtype (implicated in the VEGFB and PGF studies above) whereas soluble FLT1 is the inert decoy subtype, which potently inhibits VEFGA-induced angiogenesis by sequestering VEGFA and blocking its activity [41]. Thus, FLT1 may have additional, indirect effects on angiogenesis by regulating the bioavailability of VEGFA in the brain. Notably, when evaluating FLT1 isoforms in the present analysis, only isoforms encoding membrane-bound FLT1 proteins showed associations with cognition and $\beta$-amyloid and tau burden, suggesting that the membrane-bound form of FLT1 may be particularly relevant to AD dementia. The isoform-specific effects of FLT1 at the gene and protein level highlight the need for a more comprehensive assessment of VEGF ligand and receptor activity during aging and disease. Our observations support FLT1 as a signaling hub for AD-related alterations.

Given the cross-sectional nature of the gene expression measurement in the present analyses, it is unclear whether the observed effects are a cause or consequence of neuropathology. Both $\gamma$-secretase [42] and $\beta$-secretase [43] (which cleave the amyloid precursor protein) are known regulators of FLT1, particularly membrane-bound forms of FLT1 [44], suggesting that there is a common upstream regulator of both $\mathrm{AD}$ pathology and $V E G F$ receptor genes. However, FLT1 contains structural hypoxic response elements which enable it to be directly upregulated in hypoxia- ischemia [45], leaving open the possibility that the observed gene expression differences are in response to injury downstream of pathology. Regardless of the causal or noncausal role, the present results suggest there are changes in the VEGFB-FLT1 signaling axis during the development $\mathrm{AD}$ pathology which are relevant to the cognitive progression of the disease.

In addition to $F L T 1$, we observed comparable associations with FLT4, whereby higher levels of prefrontal cortex expression were (1) observed among participants with $\mathrm{AD}$ dementia compared with those with normal cognition, (2) associated with faster rates of cognitive decline in the years preceding death, and (3) associated with greater amyloid and tau burden at autopsy. Similar to FLT1, FLT4 contains structural elements that are directly responsive to hypoxia [46], suggesting a potential common pathway. FLT4 appears to be required for adult neurogenesis [47] and the upregulation of lymphogenesis, but not angiogenesis in the brain [48]. Given these beneficial effects, the negative association of FLT4 observed here may reflect a cellular response to $A D$ neuropathology rather than acting as a driver of neuropathology and neural injury.

This study has multiple strengths, including the wellcharacterized cohorts assessed, the measurement of VEGF ligand and receptor genes in postmortem brain tissue, and the availability of comprehensive longitudinal cognitive data. However, the measurement of gene expression in postmortem brain tissue precludes interpretation about causality or directionality from our analyses and leaves open the possibility that observed effects could be a cause, a consequence, or simply a co-occurrence with disease. Further, brain homogenates were leveraged for RNA analysis and include cell-type differences across individuals that could confound results. We attempted to control for these effects by adjusting for cell-type-specific gene expression markers and the results from the adjusted models did not change the interpretation of our main findings. However, such statistical adjustments may be insufficient to fully correct for cell-specific effects. In addition, only binary variables of the presence or absence of micro-infarcts were available for analysis, and we had no biochemical measure of hypoperfusion in the brain, limiting our ability to draw definitive conclusions about all types of ischemic damage in the brain. Finally, the sample evaluated included a highly educated, homogenous cohort of primarily European ancestry that may not generalize to other populations.

In summary, we examined associations across the VEGF family of genes with cognition, $\mathrm{AD}$ dementia, and $\mathrm{AD}$ pathology, and identified genes in the FLT4 and FLT1 signaling pathways as being particularly relevant. Future work will explore how VEGF family protein expression associates with cognition and $\mathrm{AD}$ pathology to 
better understand how changes in VEGF transcription and translation relate to neurodegenerative disease.

Acknowledgements This research was supported in part by K01AG049164, R01-AG059716, R21-AG05994, K12-HD043483, K24-AG046373, HHSN311201600276P, S10-OD023680, R01AG034962， R01-NS100980， R01-AG056534， P30-AG010161, R01-AG15819, R01-AG17917, U01-AG46152, UL1-TR000445, and the Vanderbilt Memory \& Alzheimer's Center. The results published here are in part based on data obtained from the AMP-AD Knowledge Portal (https://doi.org/10.7303/syn2580853). MSBB data were generated from postmortem brain tissue collected through the Mount Sinai VA Medical Center Brain Bank and were provided by Dr Eric Schadt from Mount Sinai School of Medicine (U01-AG046170). MayoRNAseq data were provided by the following sources: The Mayo Clinic Alzheimers Disease Genetic Studies, led by Dr Nilufer Ertekin-Taner and Dr Steven G. Younkin, Mayo Clinic, Jacksonville, FL using samples from the Mayo Clinic Study of Aging, the Mayo Clinic Alzheimers Disease Research Center, and the Mayo Clinic Brain Bank. Data collection was supported through funding by NIA grants P50 AG016574, R01 AG032990, U01 AG046139, R01 AG018023, U01 AG006576, U01 AG006786, R01 AG025711, R01 AG017216, R01 AG003949, NINDS grant R01 NS080820, CurePSP Foundation, and support from Mayo Foundation. Study data includes samples collected through the Sun Health Research Institute Brain and Body Donation Program of Sun City, Arizona. The Brain and Body Donation Program is supported by the National Institute of Neurological Disorders and Stroke (U24 NS072026 National Brain and Tissue Resource for Parkinsons Disease and Related Disorders), the National Institute on Aging (P30 AG19610 Arizona Alzheimers Disease Core Center), the Arizona Department of Health Services (contract 211002, Arizona Alzheimers Research Center), the Arizona Biomedical Research Commission (contracts 4001, 0011, 05-901, and 1001 to the Arizona Parkinson's Disease Consortium), and the Michael J. Fox Foundation for Parkinsons Research.

\section{Compliance with ethical standards}

Conflict of interest The authors declare that they have no conflict of interest

Publisher's note: Springer Nature remains neutral with regard to jurisdictional claims in published maps and institutional affiliations.

Open Access This article is licensed under a Creative Commons Attribution 4.0 International License, which permits use, sharing, adaptation, distribution and reproduction in any medium or format, as long as you give appropriate credit to the original author(s) and the source, provide a link to the Creative Commons license, and indicate if changes were made. The images or other third party material in this article are included in the article's Creative Commons license, unless indicated otherwise in a credit line to the material. If material is not included in the article's Creative Commons license and your intended use is not permitted by statutory regulation or exceeds the permitted use, you will need to obtain permission directly from the copyright holder. To view a copy of this license, visit http://creativecommons. org/licenses/by/4.0/.

\section{References}

1. Ruiz de Almodovar C, Lambrechts D, Mazzone M, Carmeliet P. Role and therapeutic potential of VEGF in the nervous system. Physiol Rev. 2009;89:607-48.
2. Garcia KO, Ornellas FLM, Martin PKM, Patti CL, Mello LE, Frussa-Filho R, et al. Therapeutic effects of the transplantation of VEGF overexpressing bone marrow mesenchymal stem cells in the hippocampus of murine model of Alzheimer's disease. Front Aging Neurosci. 2014;6:6-30.

3. Hohman TJ, Bell SP, Jefferson AL. The Role of vascular endothelial growth factor in neurodegeneration and cognitive decline: exploring interactions with biomarkers of Alzheimer disease. JAMA Neurol. 2015;72:520-9.

4. Huang L, Jia J, Liu R. Decreased serum levels of the angiogenic factors VEGF and TGF- $\beta 1$ in Alzheimer's disease and amnestic mild cognitive impairment. Neurosci Lett. 2013;550:60-3.

5. Tarkowski E, Issa R, Sjögren M, Wallin A, Blennow K, Tarkowski $\mathrm{A}$, et al. Increased intrathecal levels of the angiogenic factors VEGF and TGF- $\beta$ in Alzheimer's disease and vascular dementia. Neurobiol Aging. 2002;23:237-43.

6. Chiappelli M, Borroni B, Archetti S, Calabrese E, Corsi MM, Franceschi M, et al. VEGF gene and phenotype relation with Alzheimer's disease and mild cognitive impairment. Rejuvenation Res. 2006;9:485-93.

7. Miners JS, Palmer JC, Love S. Pathophysiology of Hypoperfusion of the Precuneus in Early Alzheimer's Disease. Brain Pathol. 2016;26:533-41.

8. Miners JS, Schulz I, Love S. Differing associations between A $\beta$ accumulation, hypoperfusion, blood-brain barrier dysfunction, and loss of PDGFRB pericyte marker in the precuneus and parietal white matter in Alzheimer's disease. J Cereb Blood Flow Metab. 2018;38:103-15.

9. Thomas T, Miners S, Love S. Post-mortem assessment of hypoperfusion of cerebral cortex in Alzheimer's disease and vascular dementia. Brain. 2015;138(Pt 4):1059-69.

10. Tang H, Mao X, Xie L, Greenberg DA, Jin K. Expression level of vascular endothelial growth factor in hippocampus is associated with cognitive impairment in patients with Alzheimer's disease. Neurobiol Aging. 2013;34:1412-5.

11. Provias J, Jeynes B. Reduction in vascular endothelial growth factor expression in the superior temporal, hippocampal, and brainstem regions in Alzheimer's disease. Current Neurovasc Res. 2014;11:202-9.

12. Lee S, Tong M, Hang S, Deochand C, de la Monte S. CSF and brain indices of insulin resistance, oxidative stress, and neuroinflammation in early versus late Alzheimer's disease. J Alzheimer's Dis Parkinsonism. 2013;3:128.

13. Guo L-H, Alexopoulos P, Perneczky R. Heart-type fatty acid binding protein and vascular endothelial growth factor: cerebrospinal fluid biomarker candidates for Alzheimer's disease. Eur Arch Psychiatry Clin Neurosci. 2013;263:553-60.

14. Spuch C, Antequera D, Portero A, Orive G, Hernández RM, Molina JA, et al. The effect of encapsulated VEGF-secreting cells on brain amyloid load and behavioral impairment in a mouse model of Alzheimer's disease. Biomaterials. 2010;31:5608-18.

15. Cao L, Jiao X, Zuzga DS, Liu Y, Fong DM, Young D, et al. VEGF links hippocampal activity with neurogenesis, learning and memory. Nat Genet. 2004;36:827.

16. Li X, Lee C, Tang Z, Zhang F, Arjunan P, Li Y, et al. VEGF-B: a survival, or an angiogenic factor? Cell Adh Migr. 2009;3:322-7.

17. Li Y, Zhang F, Nagai N, Tang Z, Zhang S, Scotney P, et al. VEGF-B inhibits apoptosis via VEGFR-1-mediated suppression of the expression of $\mathrm{BH} 3$-only protein genes in mice and rats. $\mathrm{J}$ Clin Investig. 2008;118:913.

18. Han J, Calvo C-F, Kang TH, Baker KL, Park J-H, Parras C, et al. Vascular endothelial growth factor receptor 3 controls neural stem cell activation in mice and humans. Cell Rep. 2015;10:1158-72.

19. Bennett DA, Schneider JA, Arvanitakis Z, Wilson RS. Overview and findings from the religious orders study. Curr Alzheimer Res. 2012;9:628. 
20. Bennett DA, Schneider JA, Buchman AS, Barnes LL, Boyle PA, Wilson RS. Overview and findings from the Rush Memory and Aging Project. Curr Alzheimer Res. 2012;9:646.

21. Bennett DA, Buchman AS, Boyle PA, Barnes LL, Wilson RS, Schneider JA. Religious Orders Study and Rush Memory and Aging Project. J Alzheimer Dis. 2018;64:S161-89.

22. Wilson RS, Boyle PA, Yu L, Barnes LL, Sytsma J, Buchman AS, et al. Temporal course and pathologic basis of unawareness of memory loss in dementia. Neurology. 2015;85:984-91.

23. Mostafavi S, Gaiteri C, Sullivan SE, White CC, Tasaki S, Xu J, et al. A molecular network of the aging human brain provides insights into the pathology and cognitive decline of Alzheimer's disease. Nat Neurosci. 2018;21:811-9.

24. Amador-Ortiz C, Lin WL, Ahmed Z, Personett D, Davies P, Duara R, et al. TDP-43 immunoreactivity in hippocampal sclerosis and Alzheimer's disease. Ann Neurol. 2007;61:435-45.

25. Arvanitakis Z, Capuano AW, Leurgans SE, Buchman AS, Bennett DA, Schneider JA. The relationship of cerebral vessel pathology to brain microinfarcts. Brain Pathol. 2017;27:77-85.

26. Arvanitakis Z, Leurgans SE, Barnes LL, Bennett DA, Schneider JA. Microinfarct pathology, dementia, and cognitive systems. Stroke. 2011;42:722-7.

27. Boyle PA, Yu L, Nag S, Leurgans S, Wilson RS, Bennett DA, et al. Cerebral amyloid angiopathy and cognitive outcomes in community-based older persons. Neurology. 2015;85:1930-6.

28. Buchman AS, Leurgans SE, Nag S, Bennett DA, Schneider JA. Cerebrovascular disease pathology and parkinsonian signs in old age. Stroke. 2011;42:3183-9.

29. Love S, Chalmers K, Ince P, Esiri M, Attems J, Jellinger K, et al. Development, appraisal, validation, and implementation of a consensus protocol for the assessment of cerebral amyloid angiopathy in post-mortem brain tissue. Am J Neurodegener Dis. 2014;3:19.

30. Schneider J, Wilson R, Cochran E, Bienias J, Arnold SE, Evans $\mathrm{D}$, et al. Relation of cerebral infarctions to dementia and cognitive function in older persons. Neurology. 2003;60:1082-8.

31. Schneider JA, Boyle PA, Arvanitakis Z, Bienias JL, Bennett DA. Subcortical infarcts, Alzheimer's disease pathology, and memory function in older persons. Ann Neurol. 2007;62:59-66.

32. Allen M, Carrasquillo MM, Funk C, Heavner BD, Zou F, Younkin CS, et al. Human whole genome genotype and transcriptome data for Alzheimer's and other neurodegenerative diseases. Sci Data. 2016;3:160089.

33. Allen M, Wang X, Burgess JD, Watzlawik J, Serie DJ, Younkin $\mathrm{CS}$, et al. Conserved brain myelination networks are altered in Alzheimer's and other neurodegenerative diseases. Alzheimers Dement. 2018;14:352-66.

34. Wang M, Beckmann ND, Roussos P, Wang E, Zhou X, Wang Q, et al. The Mount Sinai cohort of large-scale genomic, transcriptomic and proteomic data in Alzheimer disease. Sci Data. 2018;5:180185.

35. Murakami M, Iwai S, Hiratsuka S, Yamauchi M, Nakamura K, Iwakura Y, et al. Signaling of vascular endothelial growth factor receptor-1 tyrosine kinase promotes rheumatoid arthritis through activation of monocytes/macrophages. Blood. 2006;108:1849-56.

36. Harris R, Miners JS, Allen S, Love S. VEGFR1 and VEGFR2 in Alzheimer's Disease. J Alzheimers Dis. 2018;61:741-52.

37. Ryu JK, Cho T, Choi HB, Wang YT, McLarnon JG. Microglial VEGF receptor response is an integral chemotactic component in Alzheimer's disease pathology. J Neurosci. 2009;29:3-13.

38. Sun Y, Jin K, Childs JT, Xie L, Mao XO, Greenberg DA. Vascular endothelial growth factor-B (VEGFB) stimulates neurogenesis: evidence from knockout mice and growth factor administration. Dev Biol. 2006;289:329-35.

39. Dhondt J, Peeraer E, Verheyen A, Nuydens R, Buysschaert I, Poesen K, et al. Neuronal FLT1 receptor and its selective ligand VEGF-B protect against retrograde degeneration of sensory neurons. FASEB J. 2011;25:1461-73.

40. Anisimov A, Leppänen V-M, Tvorogov D, Zarkada G, Jeltsch M, Holopainen T, et al. The basis for the distinct biological activities of vascular endothelial growth factor receptor-1 ligands. Sci Signal. 2013;6:ra52.

41. Olsson A-K, Dimberg A, Kreuger J, Claesson-Welsh L. VEGF receptor signalling? In control of vascular function. Nat Rev Mol Cell Biol. 2006;7:359.

42. Boulton ME, Cai J, Grant MB, Zhang Y. Gamma-secretase regulates VEGFR-1 signalling in vascular endothelium and RPE. Adv Exp Med Biol. 2008;613:313-9.

43. Cai J, Qi X, Kociok N, Skosyrski S, Emilio A, Ruan Q, et al. Beta-Secretase (BACE1) inhibition causes retinal pathology by vascular dysregulation and accumulation of age pigment. EMBO Mol Med. 2012;4:980-91.

44. Rahimi N, Golde TE, Meyer RD. Identification of ligand-induced proteolytic cleavage and ectodomain shedding of VEGFR-1/FLT1 in leukemic cancer cells. Cancer Res. 2009;69:2607-14.

45. Ulyatt C, Walker J, Ponnambalam S. Hypoxia differentially regulates VEGFR1 and VEGFR2 levels and alters intracellular signaling and cell migration in endothelial cells. Biochem Biophys Res Commun. 2011;404:774-9.

46. Liu J-L, Xia X-B, Xu H-Z. Expression of FLT4 in hypoxiainduced neovascular models in vitro and in vivo. Int $\mathrm{J}$ Ophthalmol. 2011;4:26-9.

47. Calvo C-F, Fontaine RH, Soueid J, Tammela T, Makinen T, AlfaroCervello C, et al. Vascular endothelial growth factor receptor 3 directly regulates murine neurogenesis. Genes Dev. 2011;25:831-44.

48. Zhang L, Zhou F, Han W, Shen B, Luo J, Shibuya M, et al. VEGFR-3 ligand-binding and kinase activity are required for lymphangiogenesis but not for angiogenesis. Cell Res. 2010;20:1319. 